\title{
Capacidade para o trabalho entre trabalhadores do Brasil
}

\author{
Work ability among workers in Brazil
}

\author{
Técia Maria Santos Carneiro e Cordeiro', Tânia Maria de Araújo'
}

\begin{abstract}
RESUMO | Contexto: A capacidade para o trabalho é um conceito que envolve condições físicas, mentais e sociais, além de ser um construto subjetivo que poderá acompanhar trabalhadores em nível individual ou coletivo. Objetivos: Descrever o perfil das produções científicas sobre capacidade para o trabalho entre trabalhadores do Brasil e identificar a prevalência e os fatores associados à capacidade para o trabalho. Métodos: Trata-se de uma revisão sistemática realizada na Biblioteca Virtual de Saúde e na Scopus. Os estudos selecionados foram aqueles publicados de 1996 a 2013, tendo como população-alvo os trabalhadores do Brasil e que utilizaram o índice de capacidade para o trabalho (ICT). Resultados: As produções científicas se concentraram nas áreas da Saúde Pública e de Enfermagem, nas regiões Sudeste e Sul do país e com trabalhadores de Enfermagem e do setor de produção. A prevalência da capacidade para o trabalho inadequada $(0,0$ a $81,2 \%)$ variou entre as diferentes categorias profissionais. Os fatores associados à capacidade para o trabalho foram os individuais, relacionados à saúde, e aqueles referentes às condições de trabalho ambiental e organizacional. Conclusões: Com base nos resultados e considerando a capacidade para o trabalho inadequada, um agravo evitável no campo da saúde do trabalhador, são necessárias ações e estratégias nos ambientes de trabalho para prevenção e promoção da saúde dos trabalhadores brasileiros. Também são necessários maiores investimentos em pesquisas longitudinais e de intervenção com diversos grupos de trabalhadores do Brasil.
\end{abstract}

Palavras-chave I saúde do trabalhador; avaliação da capacidade de trabalho; envelhecimento prematuro.

ABSTRACT | Context: The ability to work involves physical, mental, and social conditions, as well as being a subjective construct that can be related to workers at the individual or collective level. Objectives: To describe the profile of scientific productions on the ability to work among Brazilian workers and to identify the prevalence and factors associated with the ability to work. Methods: This is a systematic review carried out in the Virtual Health Library and in Scopus. Studies selected were those published from 1996 to 2013 , whose target population was Brazilian workers and which used the work ability index (WAI). Results: The scientific productions focused on the fields of Public Health and Nursing, in the Southeast and South regions of the country, and on workers in the Nursing and production fields. The prevalence of inadequate work abilities ( 0.0 to $81.2 \%)$ varied among the different professional categories. Factors associated with the ability to work were individual, health-related, and those related to environmental and organizational working conditions. Conclusions: Based on the results and considering inadequate work ability, which is an avoidable problem in the worker's health field, actions and strategies are necessary for prevention and promotion of health among Brazilian workers. Further investments are also needed in longitudinal and interventional studies with various groups of Brazilian workers.

Keywords I occupational health; work capacity evaluation; aging, premature. 


\section{INTRODUÇÃO}

A capacidade para o trabalho refere-se à autopercepção do indivíduo sobre a sua própria saúde, trabalho e estilo de vida, sendo um construto multidimensional e versátil por envolver pré-condições físicas, mentais e sociais. Esse conceito de capacidade para o trabalho, para alguns especialistas, depende do ponto de vista considerado, mas, em geral, trata de um equilíbrio entre o trabalho e os recursos pessoais, sendo que todos os fatores que $o$ afetam mudam continuamente. Baseia-se na perspectiva de, com base em acompanhamento periódico, melhorar a vida no trabalho por um período mais longo ${ }^{1,2}$.

Para avaliar a capacidade para o trabalho, foi desenvolvido o índice de capacidade para o trabalho (ICT) a partir de pesquisas na Finlândia, na década de 1980. A tradução para uso no Brasil ocorreu em 1996. O ICT é destinado ao uso em serviços de saúde ocupacional, e possibilita pesquisas no âmbito científico por ser preciso e confiável. Os resultados são reproduzíveis e podem ser utilizados para pesquisas e/ou acompanhamentos nos níveis individuais e coletivos, além da possibilidade de avaliar tanto a capacidade funcional do trabalhador quanto os fatores associados a ela.É dividido em sete dimensões, que são mensuradas em um escore que pode variar de 7 a 49 pontos e que são classificados em 4 categorias (baixa, moderada, boa e ótima) ${ }^{3}$. Existem ainda pesquisadores que dicotomizam esses escores em capacidade para o trabalho inadequada ( $\leq 36$ pontos) e capacidade para o trabalho adequada ( $\geq 37$ pontos) $)^{4}$.

Segundo Tuomi et al. ${ }^{5}$, o ICT refere-se a "Quão bem está, ou estará, um(a) trabalhador(a) presentemente ou num futuro próximo, e quão capaz ele ou ela podem executar seu trabalho, em função das exigências, de seu estado de saúde e de suas capacidades físicas e mentais".

A avaliação da capacidade para o trabalho tem se mostrado relevante para o campo da saúde do trabalhador devido aos impactos na força de trabalho, gerados pelo envelhecimento precoce e também pela transição demográfica, com o envelhecimento da população. Essa avaliação subsidia ações e estratégias para manutenção e/ou promoção da capacidade para o trabalho, visando melhores condições de trabalho e de vida saudáveis.

Apesar dos vários indicadores que apontam maior longevidade da população trabalhadora e a sua maior permanência no mercado de trabalho, há poucas investigações com foco em aspectos relacionados à capacidade para o trabalho no Brasil. Dessa forma, justifica-se este estudo por considerar a escassez de revisões sistemáticas acerca da capacidade para o trabalho entre trabalhadores brasileiros e a relevância de se utilizar um instrumento com desempenho satisfatório para mensurar a capacidade para o trabalho e, em consequência, subsidiar ações e estratégias para prevenção de agravos e promoção da saúde dos trabalhadores.

Este estudo tem como objetivos descrever o perfil das produções científicas sobre capacidade para o trabalho entre trabalhadores do Brasil e identificar a prevalência e os fatores associados à capacidade para o trabalho.

\section{MÉTODOS}

Trata-se de uma revisão sistemática, a qual permite conduzir investigações de dados já publicados. Foram adotadas as diretrizes do Preferred Reporting Items for Systematic Reviews and Meta-Analyses (PRISMA) ${ }^{6}$.

\section{CRITÉRIOS DE ELEGIBILIDADE DO CORPUS DOCUMENTAL}

Os critérios de inclusão adotados para seleção dos artigos analisados foram:

- estudos publicados no período de 1996 a 2013;

- estudos publicados nosidiomas português, inglês e espanhol;

- estudos com população-alvo de trabalhadores do Brasil;

- estudos com uso do ICT para avaliar a capacidade para o trabalho.

Os critérios de exclusão foram:

- estudos que utilizaram o ICT incompleto;

- estudos de revisão;

- estudos de validação de instrumento.

Não houve restrições para os demais tipos de estudos. Considerou-se a busca a partir de 1996 por se tratar do ano de tradução do instrumento no Brasil.

\section{ESTRATÉGIA DE BUSCA E SELEÇÃO}

A busca de dados foi conduzida na Biblioteca Virtual de Saúde (BVS) por consolidar várias bases de dados (SciELO, BDENF, LILACS, MEDLINE, IBECS, Biblioteca Cochrane) e na Scopus, por agrupar resumos de bases internacionais. Foram utilizadas as palavras "capacidade para o trabalho" e "work ability" e os descritores "avaliação da capacidade de trabalho" 
e "work ability evaluation". As palavras foram combinadas com os descritores por meio do operador booleano "OR"; optou-se por essa combinação pelo aumento no universo da busca. $\mathrm{Na}$ BVS, a pesquisa restringiu-se a: "capacidade para o trabalho" OR "avaliação da capacidade de trabalho", ano calendário 19962013; idiomas: português, inglês e espanhol; texto completo e artigos. Na Scopus, a busca se restringiu a: "work ability" OR "work ability evaluation", ano calendário 1996-2013, artigos, filiação institucional (universidades brasileiras) e idiomas: português, inglês e espanhol. Após seleção na Scopus, foi realizada a busca de cada artigo nos sites dos periódicos. A busca dos artigos foi finalizada em dezembro de 2014.

$\mathrm{Na}$ primeira análise, selecionaram-se os artigos pelas informações contidas no título e nas palavras-chave disponíveis na base de dados. Posteriormente, foi feita a leitura dos resumos e dos artigos completos para avaliação dos critérios de inclusão da revisão proposta. Os critérios de elegibilidade foram aplicados tanto durante a busca de dados quanto na seleção dos artigos. O processo de busca foi realizado por avaliador competente na área em estudo.

\section{AVALIAÇÃO DA QUALIDADE METODOLÓGICA}

A avaliação da qualidade metodológica e dos riscos de viés dos artigos foi realizada pela lista de critérios metodológicos elaborada por Proper et al. ${ }^{7}$ com base nas orientações adaptadas da Cochrane ${ }^{8}$. A lista é composta de nove itens que se referem à qualidade do objetivo, à seleção da amostra, aos critérios de elegibilidade, à participação dos sujeitos, ao instrumento de coleta, à coleta de dados, ao tratamento estatístico, às limitações e às conclusões.

A pontuação varia de 0 a 9 pontos, sendo que estudos populacionais variam até 8 pontos por não se enquadrarem na aleatoriedade da amostra. Para cada critério, adotou-se a classificação de Mascarenhas e Fernandes ${ }^{8}$ : + (atende totalmente ao item); +/-(atende parcialmente); - (não atende); NSA (não se aplica). O escore da avaliação metodológica é pontuado apenas pelo + . Os estudos foram considerados de elevada qualidade quando apresentavam 50\% dos itens positivos; e de baixa qualidade quando a pontuação obtida foi menor do que $50 \%$.

\section{COLETA DE DADOS}

Para a coleta de dados, foi elaborada uma ficha com as seguintes informações: identificação, título, periódico, ano de publicação, idioma de publicação, base de dados, instituição/ universidade responsável pela produção, objetivo, tipo de estudo e análise, confiabilidade do instrumento, pontos de corte da escala do ICT, locus da pesquisa, população de estudo, classificação brasileira de ocupações, prevalência da capacidade para o trabalho, fatores associados à capacidade para o trabalho, aspectos relevantes e principais considerações. Os fatores associados foram considerados entre os estudos de análise inferencial que alcançaram resultados estatisticamente significantes de acordo com os critérios estatísticos adotados em cada estudo.

\section{ANÁLISE DOS DADOS}

Os artigos foram analisados através da bibliometria com suporte do Microsoft Office Excel 2007 a partir da estatística descritiva com frequência absoluta e relativa, com o intuito de determinar as características do corpus documental desta revisão. Para identificar a prevalência da capacidade para o trabalho em adequada (ICT $\geq 37$ pontos) nos artigos selecionados que apresentavam os resultados em quatro categorias (baixa, moderada, boa e ótima), foram somadas as frequências das categorias boa e ótima; e para a categoria inadequada (ICT $\leq 36$ pontos), foram somadas as categorias baixa e moderada. Dessa forma, não foram analisadas as prevalências dos artigos que apresentaram os resultados do ICT com uso de escala contínua. Os fatores associados à capacidade para o trabalho foram apresentados para os respectivos estudos e pela categoria profissional.

\section{RESULTADOS}

\section{SELEÇÃO E CARACTERÍSTICAS DO CORPUS DOCUMENTAL}

Foram encontrados 2.185 artigos, excluídos 2.145 por não atenderem aos critérios de elegibilidade, sendo que 40 foram incluídos na revisão sistemática (Figura 1). A Tabela 1 apresenta as características do corpus documental desta revisão. O período com maior publicação foi de 2009 a 2013 e a instituição/universidade responsável pelo maior número de publicações foi a Universidade de São Paulo (USP). As principais áreas de publicação dos periódicos foram Saúde Pública e Enfermagem. Houve apenas um estudo quase-experimental e um caso-controle; nove verificaram a consistência interna do ICT por meio do alfa de Cronbach com média de $0,70 \pm 0,07$, mesmo não sendo estudos de validação do instrumento. Cinco dos estudos trabalharam com a escala contínua do ICT. 
As regiões Sudeste e Sul do Brasil foram as responsáveis por $90 \%$ das publicações identificadas, com predominância dos estados de São Paulo e Rio Grande do Sul. Os profissionais-alvos das pesquisas foram, em sua maioria, os trabalhadores de enfermagem (enfermeiros, auxiliares e técnicos de enfermagem) e do setor de produção (fábricas e multinacionais de materiais escolares e de escritório, têxteis, alimentos e bebidas). De acordo a Classificação Brasileira de Ocupações ( $\mathrm{CBO}$ ), os grupos de trabalhadores com maior frequência nos estudos foram os grupos: 2 (profissionais das ciências e das artes) e 7 (trabalhadores da produção de bens e serviços industriais). Em relação ao processo de amostragem, apenas dois estudos realizaram seleção de amostra aleatória, sendo os demais, estudos de caráter censitário, com taxas de respostas que variaram de 37,9 a 96,2\%; portanto, alguns estudos registraram taxas de respostas muito baixas, abaixo do aceitável na literatura.

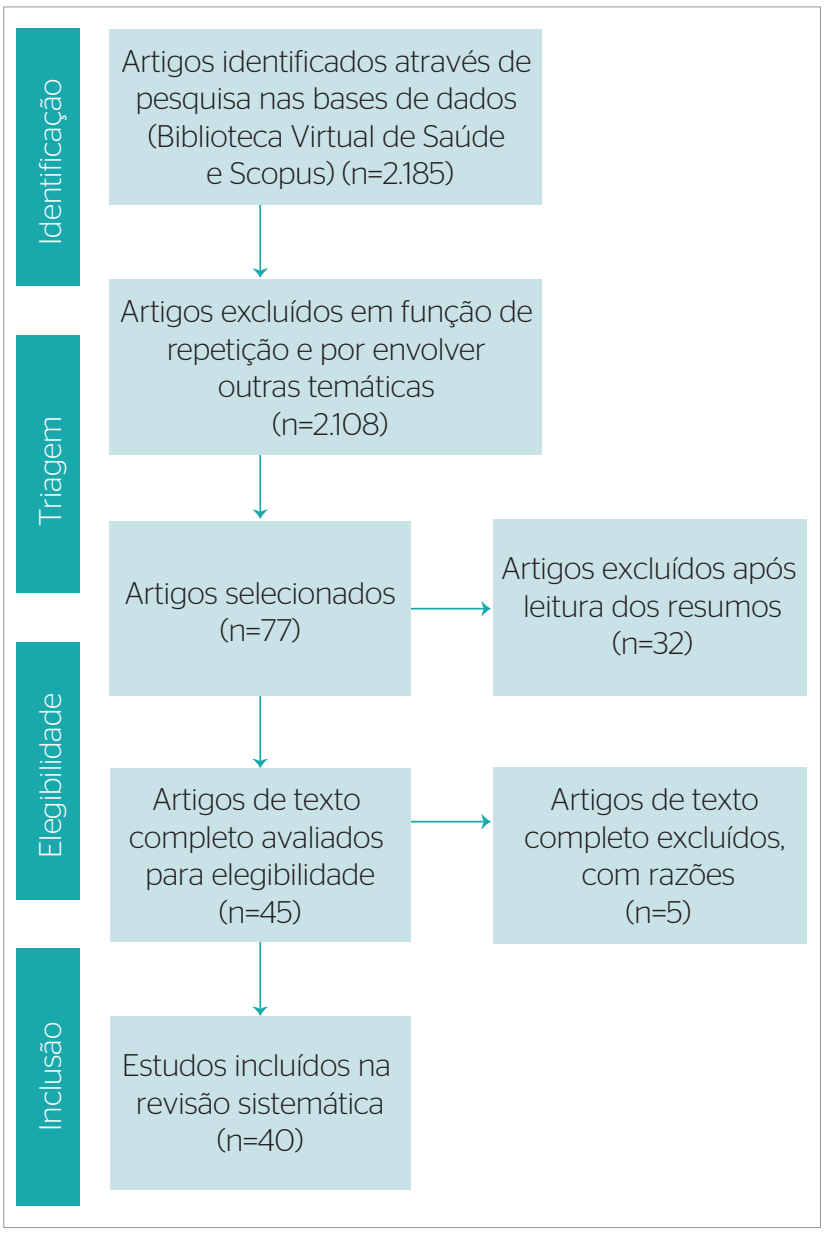

Figura1. Processo de seleção do corpus documental desta revisão.

\section{QUALIDADE METODOLÓGICA}

A avaliação da qualidade metodológica do corpus documental dos estudos identificados apresentou uma variação de cinco a nove pontos (Tabela 2). Dois estudos utilizaram critérios de aleatoriedade para selecionar a amostra. Todos os estudos utilizaram técnicas estatísticas compatíveis com o modelo de estudo adotado. Quanto às limitações, 12 artigos não apresentaram nenhuma desvantagem e vieses do estudo. Apenas um artigo apresentou o escore máximo; no entanto, os estudos foram considerados de alta qualidade, segundo o instrumento utilizado, por superar os $50 \%$ dos itens positivos estabelecidos nos parâmetros de avaliação e a maioria dos estudos $(n=22)$ apresentou escores sete e oito, embora esses artigos sejam pouco críticos ou autocríticos, já que 12 deles não apontaram suas limitações.

\section{PREVALÊNCIA DA CAPACIDADE PARA O TRABALHO}

A prevalência da capacidade para o trabalho inadequada variou entre $0,0 \%$ em estagiários da área de tecnologia da informaçã ${ }^{23}$ e $81,2 \%$ em trabalhadores portadores de lesões por esforços repetitivos e doenças osteomusculares relacionadas ao trabalho (LER/DORT) do setor de produção de uma fábrica ${ }^{15}$ (Tabela 3). Observa-se que, entre os trabalhadores de enfermagem do nível hospitalar - os quais compõem a maior parte dos grupos populacionais investigados -, a capacidade para o trabalho inadequada variou entre $11,3^{42}$ e $52,0 \%{ }^{32}$. Os estudos com trabalhadores do setor de produção apresentaram uma variação de 18,0\% entre aqueles produtores de materiais escolares e de escritório ${ }^{20}$ a $81,2 \%$ entre os portadores de LER/DORT do setor de produção de uma fábrica ${ }^{15}$.

Alguns dos estudos avaliaram a capacidade para o trabalho por gênero ${ }^{21,36,48}$, destacando-se a maior prevalência do ICT inadequado entre as mulheres. Um estudo avaliou o ICT por nível de escolaridade ${ }^{25}$, sendo observado que a capacidade para o trabalho inadequada foi maior entre os trabalhadores de nível técnico quando comparados aos de nível superior. Também foi avaliado o ICT por vínculo de trabalho, sendo que a capacidade para o trabalho inadequada foi maior entre os terceirizados quando comparados aos estagiários ${ }^{23}$, e entre os trabalhadores com vínculo permanente quando comparados com aqueles que tinham vínculo precário ${ }^{14}$. 
Tabela 1. Características do corpus documental.

Características descritivas $\quad$ n

Período de publicação

1996 a 2003

2004 a 2008

$3 \quad 7,5$

2009 a 2013

13

32,5

Instituição/universidade responsável pela produção*

Universidade de São Paulo (USP)

Universidade Federal de São Carlos (UFSCar)

18

Instituto Oswaldo Cruz (FIOCRUZ/RJ)

Universidade Federal do Rio Grande do Sul (UFRGS)

24

60,0

Outros

$18 \quad 28,1$

$6 \quad 9,4$

$4 \quad 6,3$

Área dos periódicos de publicação

Saúde Pública

Enfermagem

Fisioterapia

Outros

$4 \quad 6,3$

$32 \quad 49,9$

Idioma de publicação

Português

Inglês

$17 \quad 42,5$

Espanhol

Bases de dados

$\begin{array}{lll}\text { Biblioteca Virtual de Saúde } & 30 & 75,0\end{array}$

Scopus

$10 \quad 25,0$

Tipo de estudo

Transversal

Quase-experimental

Caso-controle

Inquérito

Não informado

11

$4 \quad 10,0$

7

Análise dos dados

\begin{tabular}{ll} 
Estatística inferencial & 34 \\
\hline
\end{tabular}

$\begin{array}{lll}\text { Estatística descritiva } & 6 & 15,0\end{array}$

Consistência interna do ICT

$\begin{array}{lll}\mathrm{Sim} & 9 & 22,5\end{array}$

$\begin{array}{lll}\text { Não } & 31 & 77,5\end{array}$

Escala do ICT

Categorizada (baixo, moderado, bom e ótimo) $\quad 22 \quad 55,0$

Dicotomizada (inadequado e adequado) $13 \quad 32,5$

Contínua $\quad 5 \quad 12,5$

Locus do estudo (estado)

2460,0

Rio Grande do Sul $\quad 6 \quad 15,0$

$\begin{array}{lll}\text { Outros } & 10 & 25,0\end{array}$

População dos estudos

Trabalhadores de Enfermagem $\quad 14 \quad 35,0$

$\begin{array}{lll}\text { Trabalhadores do setor de produção } & 7 & 17,5\end{array}$

$\begin{array}{lll}\text { Professores } & 3 & 7,5\end{array}$

$\begin{array}{lll}\text { Outros } & 16 & 40,0\end{array}$

Classificação Brasileira de Ocupações

Grupo 2 - Profissionais das Ciências e das Artes $17 \quad 42,5$

Grupo 7 - Trabalhadores da produção de bens e serviços industriais $\quad 9 \quad 22,5$

Outros $\quad 14 \quad 35,0$

Tipo de amostra

$\begin{array}{lll}\text { Populacional } & 38 & 95,0\end{array}$

Aleatória

Média da taxa de resposta dos estudos populacionais - 72,8 $\pm 16,5$ (mín. 37,9; máx. 96,2)

${ }^{*}$ Cada artigo pode ter apresentado mais de um vínculo institucional/universidade ( $\left.n=24\right)$; ICT: índice de capacidade para o trabalho. 


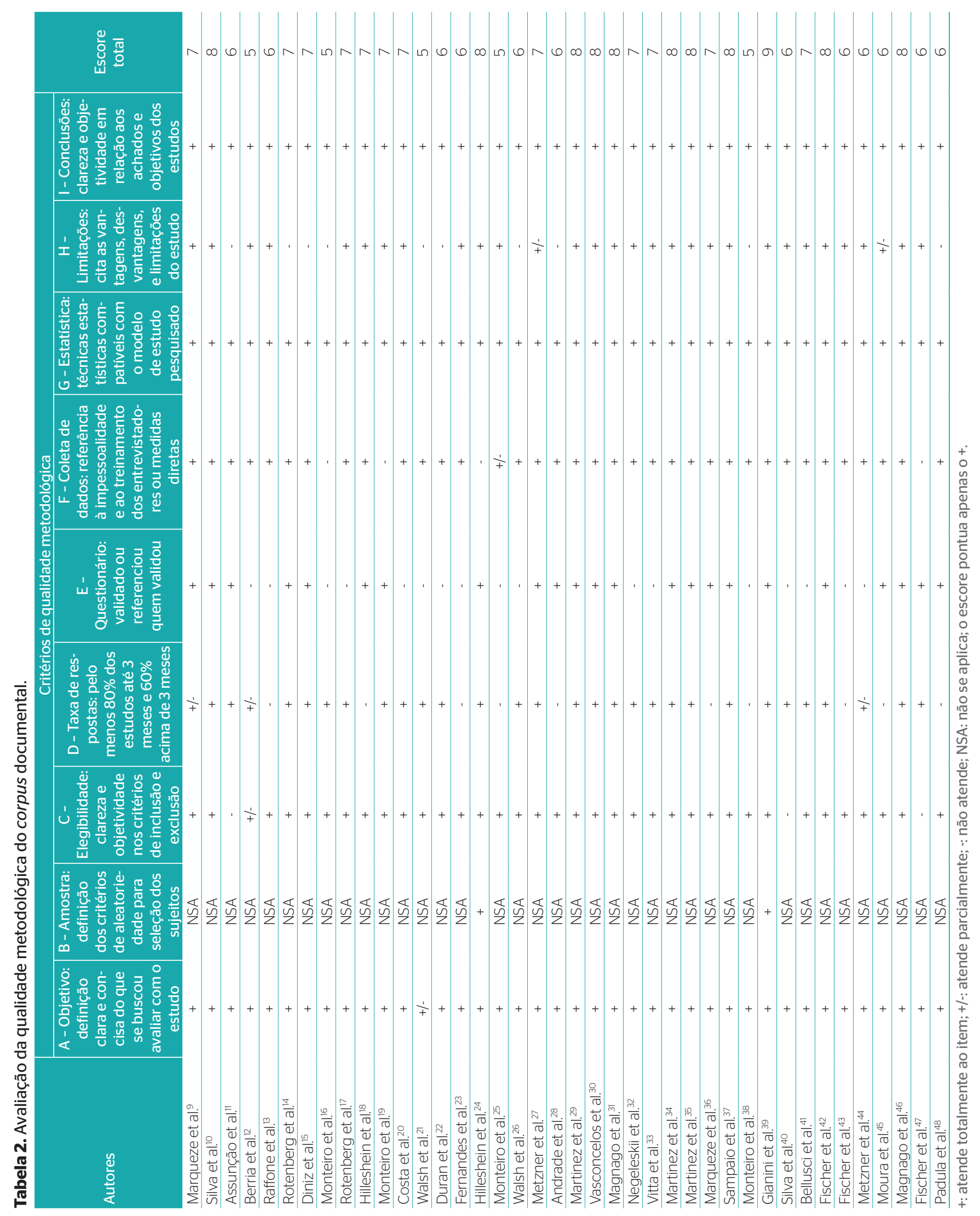


Tabela 3. Prevalência da capacidade para o trabalho e os fatores associados entre os trabalhadores do corpus documental desta revisão.

\begin{tabular}{|c|c|c|c|c|}
\hline Autor/ano & $\begin{array}{l}\text { Desenho de } \\
\text { estudo }\end{array}$ & População & CT inadequada & Fatores associados à CT \\
\hline Bellusci et al. 199941 & Transversal & Servidores forenses & $17,6 \%$ & Sexo; tempo de trabalho; cargo \\
\hline Metzner et al. $2001^{44}$ & Transversal & $\begin{array}{l}\text { Setor de produção } \\
\text { (têxteis) }\end{array}$ & - & $\begin{array}{l}\text { Tempo de trabalho na função; turno de } \\
\text { trabalho diurno }\end{array}$ \\
\hline Fischer et al. $2002^{43}$ & Transversal & $\begin{array}{l}\text { Trabalhadores de } \\
\text { enfermagem }\end{array}$ & $19,3 \%$ & Estudo descritivo, não há associação \\
\hline Walsh et al. $2004^{21}$ & Transversal & $\begin{array}{l}\text { Setor de produção } \\
\text { (multinacional) }\end{array}$ & $\begin{array}{l}\text { Homens: } 13,0 \% \\
\text { Mulheres: } 53,0 \%\end{array}$ & $\begin{array}{c}\text { Prognóstico do próprio trabalhador em } \\
\text { relação à CT; exigência física do trabalho; } \\
\text { otimismo; idade; sexo; afastamento do } \\
\text { trabalho; dor }\end{array}$ \\
\hline Duran et al. $2004^{22}$ & Transversal & Enfermagem & $13,2 \%$ & Estudo descritivo, não há associação \\
\hline Martinez et al. $2004^{34}$ & Transversal & Administrativos & $15,2 \%$ & Satisfação no trabalho; aspectos da saúde \\
\hline Raffone et al. $2005^{13}$ & Transversal & Enfermagem & $16,8 \%$ & Escolaridade; atividade física \\
\hline Monteiro et al. $2005^{25}$ & $\begin{array}{l}\text { Não } \\
\text { informado }\end{array}$ & $\begin{array}{l}\text { Setor de alta } \\
\text { tecnologia }\end{array}$ & $\begin{array}{l}\text { Técnicos: } 22,2 \% \\
\text { Nível superior: } 11,5 \%\end{array}$ & $\begin{array}{l}\text { Exigência física; número de doenças } \\
\text { diagnosticadas; absenteísmo no último } \\
\text { ano; recursos mentais; idade }\end{array}$ \\
\hline Monteiro et al. 200619 & Transversal & $\begin{array}{l}\text { Tecnologia da } \\
\text { informação }\end{array}$ & $9,2 \%$ & $\begin{array}{l}\text { Atividade física; frequência da atividade } \\
\text { física; mais de um vínculo de trabalho; sexo }\end{array}$ \\
\hline Fernandes et al. $2006^{23}$ & Transversal & $\begin{array}{l}\text { Tecnologia da } \\
\text { informação }\end{array}$ & $\begin{array}{l}\text { Estagiários: 0,0\% } \\
\text { Terceirizados: } 5,5 \%\end{array}$ & $\begin{array}{l}\text { Tempo de trabalho; atividade física; tempo } \\
\text { de deslocamento; ouvir música diariamente }\end{array}$ \\
\hline Andrade et al. $2007^{28}$ & Transversal & Limpeza hospitalar & $46,4 \%$ & Idade \\
\hline Walsh et al. $2008^{26}$ & $\begin{array}{l}\text { Não } \\
\text { informado }\end{array}$ & $\begin{array}{l}\text { Setor produ- } \\
\text { ção (material de } \\
\text { escritório) }\end{array}$ & $42,5 \%$ & $\begin{array}{l}\text { Aspectos clínicos das doenças osteo- } \\
\text { musculares relacionadas ao trabalho; } \\
\text { índice de incapacidade física }\end{array}$ \\
\hline Martinez et al. $2008^{35}$ & Transversal & Eletricitários & - & Estudo descritivo, não há associação \\
\hline Metzner et al. $2008^{27}$ & Transversal & $\begin{array}{l}\text { Setor de produção } \\
\text { (têxteis) }\end{array}$ & - & Não houve associação \\
\hline Marqueze et at. $2008^{9}$ & $\begin{array}{c}\text { Quase- } \\
\text { experimental }\end{array}$ & $\begin{array}{l}\text { Professores } \\
\text { universitários }\end{array}$ & $\begin{array}{l}\text { 1a fase: } 13,0 \% \\
\text { 2a fase: } 7,7 \%\end{array}$ & $\begin{array}{l}\text { Satisfação no trabalho; idade; sexo; mais } \\
\text { de um vínculo de trabalho }\end{array}$ \\
\hline Rotenberg et al. $2008^{17}$ & Transversal & Enfermagem & $\begin{array}{l}\text { Homens: } 25,6 \% \\
\text { Mulheres: } 40,5 \%\end{array}$ & $\begin{array}{l}\text { Homens: não houve associação } \\
\text { Mulheres: carga de trabalho total }\end{array}$ \\
\hline Monteiro et al. $2009^{16}$ & Transversal & $\begin{array}{l}\text { Trabalhadores da } \\
\text { saúde }\end{array}$ & - & Doenças musculoesqueléticas \\
\hline Marqueze et al. $2009^{36}$ & Transversal & $\begin{array}{l}\text { Professores } \\
\text { universitários }\end{array}$ & $13,0 \%$ & $\begin{array}{l}\text { Mais de um vínculo de trabalho na área } \\
\text { de saúde; satisfação no trabalho }\end{array}$ \\
\hline Rotenberg et al. $2009^{14}$ & Transversal & Enfermagem & $\begin{array}{l}\text { Vínculo permanente: } \\
\quad 46,3 \% \\
\text { Vínculo precário: 30,4\% }\end{array}$ & Trabalho precário; trabalho noturno \\
\hline Martinez et al. $2009^{29}$ & Transversal & Eletricitários & - & $\begin{array}{l}\text { Idade; tipo de trabalho; estresse no trabalho; } \\
\text { índice de massa corpórea; dependência do } \\
\text { álcool; atividade física; estado de saúde }\end{array}$ \\
\hline Sampaio et al. $2009^{37}$ & Transversal & $\begin{array}{l}\text { Trabalhadores de } \\
\text { transporte cole- } \\
\text { tivo (administra- } \\
\text { tivo, manutenção e } \\
\text { operação) }\end{array}$ & $10,7 \%$ & $\begin{array}{l}\text { Estresse no trabalho; demanda no traba- } \\
\text { Iho; atividade física }\end{array}$ \\
\hline Diniz et al. 2010'15 & Transversal & $\begin{array}{l}\text { Setor produção } \\
\text { (fábrica) com LER }\end{array}$ & $81,2 \%$ & Estudo descritivo, não há associação \\
\hline Assunção et al. 2010"1 & Inquérito & $\begin{array}{l}\text { Setor de produ- } \\
\text { ção (bebidas e } \\
\text { alimentos) }\end{array}$ & $26,2 \%$ & Sexo; idade; estado geral de saúde \\
\hline
\end{tabular}


Tabela 3. Continuação.

\begin{tabular}{|c|c|c|c|c|}
\hline Autor/ano & $\begin{array}{l}\text { Desenho de } \\
\text { estudo }\end{array}$ & População & CT inadequada & Fatores associados à CT \\
\hline Berria et al. 201112 & Transversal & Policiais & $11,9 \%$ & Não houve associação \\
\hline Hilleshein et al $2011^{18}$ & Transversal & Enfermeiros & $15,1 \%$ & $\begin{array}{l}\text { Tempo de trabalho; idade; horas de } \\
\text { sono; estado civil; uso de tabaco }\end{array}$ \\
\hline Silva et al. $2011^{10}$ & Transversal & Enfermagem & $22,8 \%$ & Não houve associação \\
\hline Vasconcelos et al. $2011^{30}$ & Transversal & Enfermagem & $40,8 \%$ & $\begin{array}{l}\text { Sexo; mais de um vínculo de trabalho; } \\
\text { condições do ambiente de trabalho; } \\
\text { número de trabalhadores no setor; tare- } \\
\text { fas repetitivas e monótonas; número de } \\
\text { doenças diagnosticadas }\end{array}$ \\
\hline Negeliskii et al. $2011^{32}$ & Transversal & Enfermeiros & $52,0 \%$ & $\begin{array}{l}\text { Tipo de unidade de trabalho (hospital, } \\
\text { aberta e adulto) }\end{array}$ \\
\hline Monteiro et al. $2011^{38}$ & Transversal & $\begin{array}{c}\text { Auxiliar de } \\
\text { enfermagem }\end{array}$ & $14,1 \%$ & $\begin{array}{l}\text { Idade; tempo de trabalho; índice de } \\
\text { massa corpórea }\end{array}$ \\
\hline
\end{tabular}

\begin{tabular}{|c|c|c|c|c|}
\hline Padula et al. $2011^{48}$ & Transversal & $\begin{array}{l}\text { Trabalhadores da } \\
\text { conservação e lim- } \\
\text { peza de uma institui- } \\
\text { ção de ensino }\end{array}$ & $\begin{array}{c}\text { Homens: 5,9\% } \\
\text { Mulheres: 26,6\% }\end{array}$ & Estudo descritivo, não há associação \\
\hline Costa et al. $2012^{20}$ & Transversal & $\begin{array}{l}\text { Setor de produção } \\
\text { (materiais escolar e } \\
\text { de escritório) }\end{array}$ & $18,0 \%$ & Idade; sexo; qualidade de vida \\
\hline Hilleshein et al. $2012^{24}$ & Transversal & Enfermeiros & $12,0 \%$ & $\begin{array}{c}\text { Satisfação com o trabalho; atividade de } \\
\text { lazer; valorização no trabalho; turno de } \\
\text { trabalho; doenças (digestivas, genituriná- } \\
\text { rias e psiquiátricas) }\end{array}$ \\
\hline Magnago et al. $2012^{31}$ & Transversal & Enfermagem & $43,3 \%$ & $\begin{array}{l}\text { Dor musculoesquelética; sexo; idade; carga } \\
\text { horária; mais de um vínculo de trabalho; } \\
\text { categoria profissional; tempo de trabalho }\end{array}$ \\
\hline Vitta et al. $2012^{33}$ & Transversal & $\begin{array}{l}\text { Trabalhadores em } \\
\text { atividades sedentá- } \\
\text { rias (empresa de for- } \\
\text { necimento de água) }\end{array}$ & $65,3 \%$ & Dor musculoesquelética \\
\hline Silva et al. $2012^{40}$ & Transversal & $\begin{array}{c}\text { Eletricitários (centro } \\
\text { de operações) }\end{array}$ & $6,9 \%$ & Satisfação no trabalho \\
\hline Fischer et al. $2012^{47}$ & Transversal & $\begin{array}{l}\text { Trabalhadores do } \\
\text { setor de alimentação } \\
\text { hospitalar }\end{array}$ & $13,8 \%$ & $\begin{array}{l}\text { Atividade física; uso de tabaco; desequi- } \\
\text { líbrio esforço-recompensa; apoio social; } \\
\text { comprometimento excessivo; doenças } \\
\text { relacionadas ao trabalho }\end{array}$ \\
\hline Gianini et al. $2013^{39}$ & Caso-controle & $\begin{array}{l}\text { Professores do } \\
\text { ensino infantil, funda- } \\
\text { mental e médio }\end{array}$ & $\begin{array}{c}\text { Casos: } 67,4 \% \\
\text { Controles: } 33,4 \%\end{array}$ & Distúrbios da voz \\
\hline Fischer et al. $2013^{42}$ & Transversal & $\begin{array}{l}\text { Trabalhadores de } \\
\text { enfermagem }\end{array}$ & $11,3 \%$ & $\begin{array}{l}\text { Idade; sexo; atividade física; índice de massa } \\
\text { corpórea; tempo de trabalho; carga horária; } \\
\text { violência no local de trabalho; percepção } \\
\text { das atividades laborais que contribuem para } \\
\text { dor e lesão; estresse no trabalho; acidente de } \\
\text { trabalho; doenças relacionadas ao trabalho }\end{array}$ \\
\hline Moura et al. $2013^{45}$ & Transversal & $\begin{array}{l}\text { Trabalhadores da } \\
\text { prefeitura do cam- } \\
\text { pus universitário }\end{array}$ & $41,0 \%$ & Estudo descritivo, não há associação \\
\hline Magnago et al. $2013^{46}$ & Transversal & $\begin{array}{c}\text { Trabalhadores de } \\
\text { enfermagem }\end{array}$ & $42,9 \%$ & Raça/cor da pele; sexo \\
\hline
\end{tabular}

CT: capacidade para o trabalho; LER: lesão por esforço repetitivo. 
Foram identificados também um estudo do tipo casocontrole e outro quase-experimental. $\mathrm{O}$ de caso-controle foi desenvolvido com professores do ensino infantil, fundamental e médio: o grupo de casos foi composto por professores que apresentaram alterações nas avaliações vocais e otorrinolaringológicas; e o grupo de controle por professores sem alterações em ambas as avaliações. A prevalência da capacidade para o trabalho inadequada foi duas vezes maior entre o grupo de casos em comparação ao de controle; e apresentou também efeito dose/reposta em relação à exposição aos distúrbios de voz independentemente do estresse, idade e acústica insatisfatória ${ }^{39}$.

O estudo do tipo quase-experimental foi desenvolvido com professores universitários em duas fases: na primeira fase foram aplicados questionários como o ICT e outros referentes aos aspectos do trabalho. Os principais resultados obtidos nessa fase, entre eles a insatisfação com a organização laboral, influenciou na intervenção do estudo, que foi a reestruturação administrativa da universidade. Na segunda fase, após dois anos, foram aplicados novamente os questionários. Os resultados finais mostraram uma tendência de melhoria da prevalência da capacidade para o trabalho, sendo que a satisfação no trabalho influenciou positivamente nessa melhora, assim como apontou a relevância da estrutura administrativa no ambiente de trabalho? .

\section{FATORES ASSOCIADOS À}

\section{CAPACIDADE PARA O TRABALHO}

De acordo com os estudos selecionados, vários fatores foram associados à prevalência da capacidade para o trabalho, influenciando em sua redução e consequente incapacidade. Os fatores por categoria profissional foram (Tabela 3):

- trabalhadores de enfermagem ${ }^{10,13,14,17,18,22,24,30-32,38,42,46}$ : sexo, idade, raça/cor da pele, escolaridade, estado conjugal, atividade física, uso de tabaco, horas de sono, atividades de lazer, índice de massa corpórea, número de doenças diagnosticadas, dor musculoesquelética, estresse no trabalho, categoria profissional, satisfação no trabalho, valorização no trabalho, trabalho precário, turno de trabalho, número de trabalhadores no setor, carga de trabalho, tempo de trabalho, condições do ambiente de trabalho, tarefas monótonas e repetitivas, mais de um vínculo de trabalho, carga horária de trabalho e violência no local de trabalho;

- trabalhadores do setor de produção ${ }^{11,15,20,21,26,27,44}$ : prognóstico do próprio trabalhador em relação à capacidade de trabalho, exigência física, otimismo, idade, sexo, afastamento do trabalho, dor, índice de capacidade física, estado geral de saúde, qualidade de vida, aspectos clínicos das doenças osteomusculares, tempo de trabalho e turno de trabalho;

- trabalhadores do setor de tecnologia ${ }^{19,23,25}$ : idade, atividade física, lazer (ouvir música), exigência física, número de doenças diagnosticadas, absenteísmo, recursos mentais, tempo de trabalho, tempo de deslocamento e mais de um vínculo de trabalho;

- trabalhadores administrativos, de atividades sedentárias eservidores forenses ${ }^{33,34,41}$ : sexo, tempo de trabalho, cargo, satisfação no trabalho, aspectos da saúde e dor musculoesquelética;

trabalhadores da limpeza hospitalar ${ }^{28}$ : idade;

- trabalhadores do setor de alimentação hospitalar ${ }^{47}$ : atividade física, uso de tabaco, desequilíbrio esforço-recompensa, apoio social, comprometimento excessivo e doença relacionada ao trabalho;

- professores ${ }^{9,36,39}$ : idade, sexo, mais de um vínculo de trabalho, satisfação no trabalho e distúrbios com a voz; trabalhadores da saúde ${ }^{16}$ : doenças musculoesqueléticas; - eletricitários ${ }^{29,40}$ : idade, atividade física, índice de massa corpórea, dependência de álcool, estado de saúde, tempo de trabalho, estresse no trabalho e satisfação no trabalho;

- trabalhadores de transporte coletivo ${ }^{37}$ : atividade física, demanda no trabalho e estresse no trabalho.

Os fatores associados à capacidade para o trabalho variaram entre as diferentes categorias profissionais; no entanto, pode-se destacar a associação entre os fatores individuais, relacionados à saúde, e aqueles referentes às condições do ambiente de trabalho e da organização laboral como fatores relacionados aos indicadores obtidos de capacidade para o trabalho.

\section{DISCUSSÃO}

O corpus documental desta revisão representa apenas 1,83\% do universo consultado, evidenciando que há uma lacuna considerável na produção científica com relação a essa temática no campo da Saúde do Trabalhador, apesar das evidências de crescente processo de envelhecimento da população trabalhadora. A capacidade para o trabalho é um fenômeno dinâmico que envolve exigências físicas, mentais, sociais e as necessidades do próprio trabalhador. Com esta revisão, percebeu-se a necessidade de uma maior atenção 
para a temática, valorizando estudos com amostra aleatória em função de serem mais efetivos na representatividade populacional, além de estudos longitudinais para acompanhar a evolução e/ou declínio da capacidade para o trabalho. Destaca-se que as regiões Norte, Nordeste e Centro-Oeste do Brasil necessitam investir em pesquisas nessa área a fim de se conhecer a capacidade funcional dos trabalhadores e, em consequência, gerar estratégias para garantir um envelhecimento ativo da população trabalhadora e prevenir aposentadorias precoces, uma vez que foram observados poucos ou nenhum estudo nessas regiões.

Além disso, as técnicas metodológicas das pesquisas precisam ser reformuladas para que tenham um retorno positivo com as ações e estratégias elaboradas a partir dos resultados. Mesmo a maioria dos estudos apresentando alta qualidade metodológica, ainda 42,5\% dos estudos apresentaram fragilidades metodológicas importantes. Assim, pode-se apontar que o viés de publicação não foi uma limitação para os artigos que compõem o corpus documental desta revisão ao considerar, também, que em três estudos não houve nenhuma associação estatisticamente significante dos fatores estudados com a capacidade para o trabalho.

A prevalência da capacidade para o trabalho inadequada diferiu entre as categorias de trabalhadores pelas características inerentes às próprias atividades laborais desempenhadas, à composição de perfil sociodemográfico dos grupos de trabalhadores investigados, às condições de trabalho, ao ambiente laboral, à organização de trabalho, aos riscos e à exposição a doenças, as quais diferem segundo objeto de trabalho, processo e organização laboral. Mas a baixa prevalência da capacidade para o trabalho inadequada, em alguns dos estudos, pode estar relacionada ao efeito do trabalhador sadio, ao considerar que os trabalhadores com a saúde comprometida podem estar afastados do mercado de trabalho, e como a maioria dos estudos analisados foi de corte transversal ${ }^{17,27,28,35,37}$, de fato se observou $o$ contexto em um ponto específico do tempo, desconsiderandose quem não estava trabalhando naquele momento.

Os fatores que apresentaram maior associação com a capacidade para o trabalho entre os estudos foram aqueles relacionados às condições de trabalho, devido aos vínculos e contratos de trabalho precários, com carga horária extensa e atuação por turnos, o que influenciava o desgaste e a consequente diminuição da capacidade. Atrelado a isso, somavam-se situações de falta de estabilidade e turno de trabalho noturno, que podem ter interferido na vida social do trabalhador ${ }^{14,24}$.
As características individuais, como a idade, estavam relacionadas ao envelhecimento cronológico do trabalhador e, associados aos demais fatores, refletem-se no envelhecimento precoce, diminuindo significativamente a capacidade para o trabalho ${ }^{18,28,38,48}$. O sexo feminino esteve associado aos piores escores do ICT em comparação ao masculino. Um dos fatores relacionados a isso pode ser a presença do trabalho doméstico, representando aumento na jornada total de trabalho das mulheres ${ }^{14,20,38,46}$.

Outra característica é a escolaridade. Foi apontado que a maior escolaridade estava associada à maior capacidade para o trabalho, evidenciando efeitos da determinação social do processo saúde-doença ${ }^{13}$, ou seja, maior acesso à informação e ao conhecimento melhora a capacidade para o trabalho. Cabe considerar também que um melhor nível de escolaridade amplia a probabilidade de inserção em postos de trabalho mais qualificados e menos agressivos à saúde, configurando situações que agudizam as desigualdades sociais nos processos de adoecimento. Portanto, esse achado pode estar relacionado não apenas ao fato de ter um nível de escolaridade maior, mas sim às questões sociais, culturais e individuais que estão atreladas à escolaridade do trabalhador.

O estilo de vida dos trabalhadores é um marcador da capacidade para o trabalho: aqueles trabalhadores que adotavam hábitos saudáveis apresentaram uma maior capacidade. Atividades de lazer, atividades físicas, o não uso de bebidas alcoólicas e do tabaco foram determinantes de uma capacidade para o trabalho adequada ${ }^{13,19,23,37,42,47}$. No entanto, os trabalhadores que apresentam uma capacidade para o trabalho reduzida possivelmente tinham baixo estímulo e autoestima para realizar atividades físicas e de lazer. Aqueles trabalhadores que não tinham hábitos saudáveis e condições laborais adequadas apresentaram maior predisposição a desenvolver doenças. As desordens musculoesqueléticas foram as doenças de maior frequência entre os estudos, por serem condições potencialmente incapacitantes e comprometerem significativamente a capacidade para o trabalho ${ }^{16,21,22,31}$.

Os fatores psicossociais também foram preditores da redução da capacidade para o trabalho. O estresse, a fadiga, a insatisfação no trabalho e a baixa autonomia determinam uma maior carga psíquica, o que reduz a capacidade. Além disso, esses fatores são pré-condições para uma satisfatória condição geral da saúde dos trabalhadores ${ }^{30,32,35-37,40,42,47}$. 
A violência no local de trabalho tem crescido nos últimos anos e constitui uma preocupação e um risco para a saúde dos trabalhadores. Dentre os fatores com destaque nos estudos realizados, foi identificado, entre os trabalhadores de enfermagem hospitalar, abuso verbal no ambiente de trabalho por parte dos pacientes, o que gera tensão e medo ${ }^{42}$. Essa situação influenciou no bem-estar mental dos trabalhadores, desencadeando a redução da capacidade para o trabalho.

Somando-se a esses fatores, um dos estudos apresentou associação da capacidade para o trabalho com a qualidade de vida em todos os domínios (físico, psicológico, social e ambiental). Apesar da qualidade de vida ser um construto amplo, percebe-se que a capacidade está associada tanto aos fatores relacionados ao trabalho quanto fora do trabalho, envolvendo várias dimensões da vida dos trabalhadores ${ }^{20}$.

Os resultados desta revisão sistemática foram semelhantes aos de outros estudos de revisão da mesma temática, os quais abordaram os fatores preditivos e determinantes da capacidade para o trabalho de trabalhadores de forma geral e de trabalhadores maiores de 65 anos de vários países ${ }^{49-51}$.

Com relação ao uso do ICT, esse instrumento mostrouse capaz de verificar o estado funcional atual dos trabalhadores por meio da avaliação e acompanhamento; além disso, pode subsidiar as ações de regulação das relações entre trabalho e saúde, e complementar os exames dos programas de saúde ocupacional ${ }^{13,14,2126,46}$. Esse instrumento também pode ser usado para avaliar a reabilitação profissional dos trabalhadores, sendo utilizado antes e depois do afastamento laboral. Os escores do ICT podem subsidiar as decisões da organização laboral. Escores baixos indicam a relevância de mudanças na organização laboral, gestão dos riscos, estilos e hábitos de vida com a implementação de programas de promoção da capacidade para o trabalho e prevenção de agravos, e intervenções nos casos de declínio da capacidade para o trabalho comprovada. Isso favorece a redução de afastamentos, aposentadorias precoces e envelhecimento precoce em decorrência do trabalho ${ }^{9,12,16,19,29,37,38,41}$.

Apesar de se tratar de uma revisão sistemática sem meta-análise, este estudo atendeu aos objetivos propostos. As limitações podem estar relacionadas ao número de artigos incluídos, devido à falta de identificação de artigos que não estavam indexados nas bases de dados utilizadas. No entanto, ainda existem poucas publicações acerca da temática com trabalhadores brasileiros; além do ICT ter sido inserido nas pesquisas no Brasil há pouco tempo e ainda estar em processo de validação para os diferentes grupos de trabalhadores, diferentemente de outros países como a Finlândia, no qual esse instrumento já faz parte das pesquisas desde a década de 1980.

Porém, o contexto das pesquisas sobre a capacidade para o trabalho no Brasil não difere dos demais países da América Latina devido à presença de características similares, como o envelhecimento progressivo da população, as mudanças decorrentes da transição demográfica e os impactos gerados na força de trabalho, fazendo com que os trabalhadores permaneçam mais tempo no mercado de trabalho. Com isso, vários aspectos como as condições precárias de trabalho, as mudanças nos processos de trabalho e na organização laboral oriundas da reestruturação produtiva devem ser considerados para avaliar periodicamente capacidade para o trabalho dos trabalhadores ativos e afastados do trabalho, visando à promoção do envelhecimento ativo, o retorno ao trabalho saudável, as mudanças no ambiente de trabalho, bem como a contribuição para a redução dos problemas sociais, econômicos e de saúde da população trabalhadora da América Latina.

\section{CONCLUSÕES}

Com esta revisão, observou-se que as produções científicas sobre a capacidade para o trabalho com o uso do ICT e com trabalhadores do Brasil se concentraram nas áreas da Saúde Pública e de Enfermagem, nas regiões Sudeste e Sul do país, e com trabalhadores de Enfermagem e do setor de produção. A qualidade metodológica dos artigos foi considerada boa. A prevalência da capacidade para o trabalho inadequada variou entre as diferentes categorias profissionais. Os fatores associados a essa prevalência foram os individuais, relacionados à saúde, e aqueles referentes às condições de trabalho ambiental e organizacional.

Ao considerar a capacidade para o trabalho inadequada, um agravo evitável no campo da saúde do trabalhador, são necessárias ações e estratégias nos ambientes de trabalho para prevenção e promoção da saúde dos trabalhadores brasileiros. Além disso, é preciso, também, maiores investimentos em pesquisas longitudinais e de intervenção com diversos grupos de trabalhadores do Brasil e dos demais países do mundo acerca dessa temática de grande relevância científica e social para o campo da saúde do trabalhador. 


\section{REFERÊNCIAS}

1. IImarinen J. Work ability: a comprehensive concept for occupational health research and prevention. Scand J Work Environ Health. 2009;35(1):1-5.

2. IImarinen J. 30 years' work ability and 20 years' age management. In: Nigard C, Savinainen M, Kirsi T, Lumme-Sandt K (Eds.). Age management during the life course: proceedings of the 4th Symposium on Work Ability. Tampere: Tampere University Press, 2011. p. 12-22.

3. Renosto A, Biz P, Hennington EA, Pattussi MP. Confiabilidade teste-reteste do índice de capacidade para o trabalho em trabalhadores metalúrgicos do Sul do Brasil. Rev Bras Epidemiol. 2009;12(2):217-25.

4. Fischer FM, Borges NS, Rotenberg L, Latorre MRDO, Soares NS, Rosa PLFS, et al. A (in)capacidade para o trabalho em trabalhadores de enfermagem. Rev Bras Med Trab. 2005;3(2):97-103.

5. Tuomi K, Ilmarinen J, Janhkola A, Katajarinne L, Tulkki A. Índice de Capacidade para o Trabalho. Traduzido por Frida Marina Fischer (coord.). São Carlos: EdUFSCar, 2005.

6. Moher D, Liberati A, Tetzlaff J, Altaman DG, The PRISMA Group. Preferred reporting items for sytematic reviews and meta-analysis: the PRISMA statement. PLoS Med. 2009;6(6):e000097.

7. Proper KI, Stall BJ, Hildebrandt VH, van der Beek AJ, van Mechelen W. Effectiveness of physical activity programs at worksites with respect to work-related outcomes. Scand J Work Environ Health. 2002;28(2):75-84

8. Mascarenhas A, Fernandes RCP. Atividades físicas de lazer e distúrbios musculoesqueléticos: revisão de literatura. Rev Baiana Saúde Pública. 2011;35(1):9-25.

9. Marquese EC, Voltz GP, Borges FNS, Moreno CRC. A 2-year follow up study among college educators. Appl Ergon. 2008;39(5):640-5.

10. Silva AA, Rotenberg L, Fischer FM. Jornadas de trabalho na enfermagem: entre necessidades individuais e condições de trabalho. Rev Saúde Pública. 2011;45(6):1117-26.

11. Assunção AA, Sampaio RF, Nascimento LMB. Agir em empresas de pequena e média dimensão para promover a saúde dos trabalhadores: o caso do setor de alimentos e bebidas. Rev Bras Fisioter. 2010;14(1):52-9.

12. Berria J, Daronco LSE, Bevilacqua LA. Aptidão motora e capacidade para o trabalho de policiais militares do batalhão de operações especiais. Salusvita. 2011;31(2):89-104.

13. Raffone AM, Hennington EA. Avaliação da capacidade funcional dos trabalhadores de enfermagem. Rev Saúde Pública. 2005;39(4):669-76.

14. Rotenberg L, Griep RH, Fischer FM, Fonseca MJM, Landsbergis P. Working at night and work ability nursing personnel: when precarious employment makes the difference. Int Arch Occup Environ Health. 2009;82(7):877-85.

15. Diniz KT, Miranda RM, Diniz ET, Moreira CH, Miranda RSS, Cabral Filho JE. Capacidade laboral dos segurados do INSS portadores de LER/DORT que retornaram ao trabalho. ConScientiae Saúde. 2010;9(4):676-83.

16. Monteiro MS, Alexandre NM, IImarien J, Rodrigues CM. Work ability and musculoskeletical disorders among workers from a public health institution. Int J Occup Saf Ergon. 2009;15(3)319-24.
17. Rotenberg L, Portela LF, Banks B, Griep RH, Fischer FM, Landsbergis P. A gender approach to work ability and its relationship to professional and domestic work hours among nursing personnel. Appl Ergon. 2008;39(5):646-52.

18. Hilleshein EF, Souza LM, Lautert L, Paz AA, Catalan VM, Teixeira MG, et al. Capacidade para o trabalho de enfermeiros de um hospital universitário. Rev Gaúcha Enferm. 2011;32(3):509-15.

19. Monteiro MI, Fernandes ACP. Capacidade para o trabalho de trabalhadores de empresa de tecnologia da informação. Rev Bras Enferm. 2006;59(5):603-8.

20. Costa CSN, Freitas EG, Mendonça LCS, Alem MER, Coury HJCG. Capacidade para o trabalho e qualidade de vida de trabalhadores industriais. Ciênc Saúde Coletiva. 2012;17(6):1635-42.

21. Walsh IAP, Corral S, Franco RN, Canetti EEF, Alem MER, Coury HJCG. Capacidade para o trabalho em indivíduos com lesões musculoesqueléticas crônicas. Rev Saúde Pública. 2004;38(2):149-56.

22. Duran ECM, Cocco MIM. Capacidade para o trabalho entre trabalhadores de enfermagem do pronto-socorro de um hospital universitário. Rev Latino-Am Enfermagem. 2004;12(1):43-9.

23. Fernandes ACP, Monteiro MI. Capacidade para o trabalho entre trabalhadores de um condomínio de empresas de alta tecnologia. Rev Bras Enferm. 2006;59(6):752-6.

24. Hilleshein EF, Lautert L. Capacidade para o trabalho, características sociodemográficas e laborais de enfermeiros de um hospital universitário. Rev Latino-Am Enfermagem. 2012;20(3):520-7.

25. Monteiro MS, IImarinen J, Gomes JR. Capacidade para o trabalho, saúde e ausência por doença de trabalhadoras de um centro de pesquisa por grupos de idade. Rev Bras Saúde Ocup. 2005;30(112):81-90.

26. Walsh IAP, Oishi J, Coury HJCG. Clinical and functional aspects of work-related musculoskeletal disorders among active workers. Rev Saúde Pública. 2008;42(1):108-16.

27. Metzner RJ, Fischer FM, Nogueira DP. Comparação da percepção de fadiga e de capacidade para o trabalho entre trabalhadores têxteis de empresas que se encontram em diferentes estágios de responsabilidade social empresarial no Estado de São Paulo, Brasil. Saúde Soc. 2008;17(4):46-55.

28. Andrade CB, Monteiro MI. Envelhecimento e capacidade para o trabalho dos trabalhadores de higiene e limpeza hospitalar. Rev Esc Enferm USP. 2007;41(2):237-44.

29. Martinez MC, Latorre MRDO. Fatores associados à capacidade para o trabalho de trabalhadores do setor elétrico. Cad. Saúde Pública. 2009;25(4):761-72.

30. Vasconcelos SP, Fischer FM, Reis AOA, Moreno CRC. Fatores associados à capacidade para o trabalho e percepção de fadiga em trabalhadores de enfermagem da Amazônia Ocidental. Rev Bras Epidemiol. 2011;14(4):688-97.

31. Magnago TSBS, Lima ACS, Prochnow A, Ceron MDS, Tavares JP, Urbanetto JS. Intensidade da dor musculoesqueletica e a (in) capacidade para o trabalho na enfermagem. Rev Latino-Am Enfermagem. 2012;20(6):1125-33.

32. Negeliskii C, Lautert L. Estresse laboral e capacidade para o trabalho de enfermeiros de um grupo hospitalar. Rev Latino-Am Enfermagem. 2011;19(3):606-13. 
33. Vitta A, Canonici AA, Conti MHS, Simeão SFAP. Prevalência e fatores associados à dor musculoesquelética em profissionais de atividades sedentárias. Fisioter Mov. 2012;25(2):273-80.

34. Martinez MC, Paraguay AIBB, Latorre MRDO. Relação entre satisfação com aspectos psicossociais e saúde dos trabalhadores. Rev Saúde Pública. 2004;38(1):55-61.

35. Martinez MC, Latorre MRDO. Saúde e capacidade para o trabalho de eletricitários do Estado de São Paulo. Ciênc Saúde Coletiva. 2008;13(3):1061-73.

36. Marqueze EC, Moreno CRC. Satisfação no trabalho e capacidade para o trabalho entre docentes universitários. Psicol Estud. 2009;14(1):75-82.

37. Sampaio RF, Coelho CM, Barbosa FB, Mancini MC, Parreira, VF. Work ability and stress in a bus transportation company in Belo Horizonte, Brazil. Ciênc Saúde Coletiva. 2009;14(1):287-96.

38. Monteiro MS, Alexandre NMC, Milani D, Fujimara F. Work capacity evaluation among nursing aides. Rev Esc Enferm USP. 2011;45(5):1177-82.

39. Gianini SPP, Latorre MRDO, Ferreira LP. Factors associated with voice disorders among teachers: a case-control study. CoDAS. 2013;25(6):566-76

40. Silva AA, Marqueze EC, Rotenberg L, Fischer FM, Moreno CRC. Shift workers in electrical systems control rooms: job satisfaction and work ability. Sleep Sci. 2012;5(2):49-55.

41. Bellusci SM, Fischer FM. Envelhecimento funcional e condições de trabalho em servidores forenses. Rev Saúde Pública. 1999;33(6):602-9.

42. Fischer FM, Martinez MC. Individual features, working conditions and work injuries are associated with work ability among nursing professionals. Work. 2013;45(4):509-17.
43. Fischer FM, Bellusci SM, Teixeira LR, Borges FNS, Ferreira R, Gonçalves $\mathrm{MBL}$, et al. Unveiling factors that contribute to functional aging among health care shift workers in São Paulo, Brazil. Exp Aging Res. 2002;28(1):73-86.

44. Metzner RJ, Fischer FM. Fadiga e capacidade para o trabalho em turnos fixos de doze horas. Rev Saúde Pública. 2001;35(6):548-53.

45. Moura AL, Reis LM, Vannuchi MTO, Haddad MCL, Domansky RC. Capacidade para o trabalho de funcionários da prefeitura de um campus universitário público. Rev Eletr Enf. 2013;15(1):130-7.

46. Magnago TSBS, Beck CLC, Greco PBT, Tavares JP, Prochnow A, Silva RM. Avaliação da capacidade para o trabalho dos trabalhadores de enfermagem de pronto-socorro. Rev Eletr Enf. 2013;15(2):523-32.

47. Fischer FM, Martinez MC. Work ability among hospital food service professionals: multiple associated variables require comprehensive intervention. Work. 2012;41(Suppl 1):3746-52.

48. Padula RS, Valente LSS, Pereira AAG, Oliveira CCP, Sperling MPR, Chiavegato L. Avaliação da capacidade para o trabalho e da aptidão cardiorrespiratória de trabalhadores saudáveis. ConScientiae Saúde. 2011;10(2):285-91.

49. Luis BL, Díaz S. Revisión bibliográfica de la capacidad funcional en trabajadores mayores de 65 años. Med Seg Trab. 2011;57(222):63-76.

50. Martinez MC, Latorre MRDO, Fischer FM. Capacidade para o trabalho: revisão de literatura. Ciênc Saúde Coletiva. 2010;15(Suppl 1):1553-61.

51. Sampaio RF, Augusto VG. Envelhecimento e trabalho: um desafio para a agenda da reabilitação. Rev Bras Fisioter. 2012;16(2):94-101.

Endereço para correspondência: Técia Maria Santos Carneiro e Cordeiro Universidade Estadual de Feira de Santana, Módulo VI, Núcleo de Epidemiologia Avenida Transnordestina, s/n - Novo Horizonte - CEP: 44036-900 - Feira de Santana (BA), Brasil - E-mail: teciamarya@yahoo.com.br 